\title{
XLIII. On the coefficient of absorption of the $\beta$ rays from uranium
}

\section{J. Arnold Crowther B.A.}

To cite this article: J. Arnold Crowther B.A. (1906) XLIII. On the coefficient of absorption of the $\beta$ rays from uranium , Philosophical Magazine Series 6, 12:70, 379-392, DOI: 10.1080/14786440609463551

To link to this article: http://dx.doi.org/10.1080/14786440609463551

$$
\text { 曲 Published online: } 16 \text { Apr } 2009 .
$$

Submit your article to this journal $₫$

Џ Article views: 2

Q View related articles $\sqsubset$

Citing articles: 20 View citing articles 5 
XLIII. On the Coefficient of Absorption of the $\beta$ Rays from Uranizm. By J. AnNold Crowther, B.A., Hutchinson Student of St. John's College, Cambridge, and Open Research Student of Emmanuel College, Cambridge*.

\section{Introduction.}

THE $\beta$ rays of radioactive substances consist of small negatively charged corpuscles travelling with a high velocity. On entering any material substance they are more or less rapidly absorbed, the intensity of the radiation decreasing, as the thickness of matter traversed is increased, very nearly in accordance with an exponential law. Thus if $l_{0}$ is the initial intensity of the radiation before entering the substance, the intensity I after passing through a thickness $d$ of the material is given by the equation

$$
\mathrm{I}=\mathrm{I}_{0} e^{-\lambda d}
$$

where $\lambda$ is the coefficient of absorption of the given substance for the particular $\beta$ radiation employed.

We may regard the absorption of the $\beta$ rays by matter as being due to collisions between the swarm of corpuscles, constituting the rays, and the particles composing the substance. From the minute size of the corpuscles, however, and their high velocity we must consider them as colliding, not with the atom or molecule of the absorbing substance as a whole, but rather as penetrating the atom, and coming into collision with the individual corpuscles of which the atom is constructed $\dagger$. The absorption of the $\beta$ rays is thus due to collision between the $\beta$ corpuscles and similar corpuscles in the atom of the absorbing medium.

On this view, therefore, the fundamental quantity in the absorption of the $\beta$ radiation is the stopping-power of the individual corpuscles in the atom, and this quantity can be immediately deduced from the coefficient of absorption. For if $\rho$ is the density of the absorbing medium, $M$ its atomic weight, and $n$ the number of corpuscles in the atom, then the number of corpuscles $\mathrm{N}$ in unit volume is given by the equation

$$
\mathrm{N}=\frac{n}{\mathbf{M}} \cdot \stackrel{\rho}{\cdot}
$$

But $n / \mathrm{M}$ is a constant, since the number of corpuscles in an atom is simply proportional to the atomic weight, and thus

\section{$\mathrm{N} \propto \rho$.}

Hence the absorption per corpusele is simply proportional to $\lambda / \rho$.

* Communicated by Prof. J. J. Thomson, F.R.S.

$\dagger$ J. J. Thomson, 'Conduction of Electricity through Gases,' p. 312 (1903). 
If the stopping-power of the corpuscle were the same for all substances, and independent of the mode of grouping of the corpuscles in the atom, we should have the ratio of the coefficient of absorption to the density of the absorbing medium a constant for all substances.

This is the "density law" of absorption, and was first propounded by Lenard* as the result of some experiments on the absorption of the cathode rays. Although these experiments included substances ranging in density from $3 \cdot 6 \times 10^{-7}$ for hydrogen at $3 \mathrm{~mm}$. pressure, to $19 \cdot 3$ for gold, and gave values of $\lambda$ varying between 0014 for the former and 55,600 for the latter, the extreme limits for the ratio $\lambda / \rho$ were only 2070 and 5610 ; thus showing that although the ratio might not be absolutely constant, it was at any rate a relation of fundamental importance.

Similar experiments were performed by Strutt $\dagger$ using the $\beta$ rays from radium, with similar results, the values obtained for $\lambda / \rho$ varying from 3.84 for card, to 7.34 for platinum. The $\beta$ radiation of radium, however, is complex, and consists of rays travelling with very different velocities. As each component ray has its own coefficient of absorption, the absorption of the bundle as a whole is not represented by any simple law, and the values obtained were therefore somewhat indeterminate.

Further experiments were therefore performed by Rutherford $\ddagger$ using uranium as the source of radiation. The $\beta$ rays from this substance are practically homogeneous, and are therefore not open to the same objection as the radium rays. The results were as follows:--n

\begin{tabular}{|c|c|}
\hline Substance. & $\lambda / \rho$. \\
\hline Glass ....... & $5 \cdot 7$ \\
\hline Mica....... & $5 \cdot 1$ \\
\hline Ebonite .......... & $5 \cdot 7$ \\
\hline Wood $\ldots . . . \ldots \ldots \ldots \ldots \ldots \ldots \ldots \ldots$ & $5 \cdot 4$ \\
\hline Cardboard ...... & $5 \cdot 3$ \\
\hline Iron $\ldots \ldots .$. & 56 \\
\hline Aluminium........ & $5 \cdot 4$ \\
\hline Copper & $7 \cdot 0$ \\
\hline Silver .... & $7 \cdot 1$ \\
\hline Lead........... & $10 \cdot 8$ \\
\hline Tin $\ldots \ldots \ldots \ldots \ldots \ldots \ldots \ldots \ldots \ldots \ldots \ldots \ldots \ldots$ & $13 \cdot 2$ \\
\hline
\end{tabular}

* Ienard, Wied. Ann. lvi. p. 255 (1895).

+ Strutt, 'Nature,' lxi. p. 539 (1900).

† Rutherford, 'Radioactivity'’ p. 114 (1904). 
An examination of this table will show that though the ratio $\lambda / \rho$ is not subject to large variations, yet there is a decided alteration as we pass from one element to another; and that consequently the stopping-power of a corpuscle is not the same in atoms of different substances.

On theoretical grounds also we might expect the nature of the arrangement of the corpuscles within the atom to have some effect upon the absorbing power. Thus Prof:. J. J. Thomson * has shown that the absorption of an atom in which all the corpuscles were rigidly connected, would be twice that of an atom containing the same number of freely moving corpuscles. It is also quite possible that the presence of so many charged corpuscles within the sprace of the atom may produce a constraint in the ether analogous to specific inductive capacity. This would affect the magnitude of the forces between the fixed and the $\beta$ corpuscles, and the extent of the alteration would depend again upon the number and arrangement of the corpuscles within the atom.

Thus differences in the value of the ratio $\lambda / \rho$ for different elements were to be expected, and it was considered desirable to make an extended series of observations upon as many elements as possible, with the object of investigating these differences and their relation to the atomic weight.

\section{Experimental.}

Uranium oxide was used as the source of the $\beta$ radiation, the homogeneity of the rays and their fairly penetrating character more than compensating for the smallness of their amount as compared with the radiation from preparations of radium. The relative intensity of the radiation before and after passing through the material under investigation was measured by the relative ionization produced in the air of a test-vessel. This is a measure of the energy of the rays, and depends not only upon the number of corpuscles but also upon their velocity. Lenard, however, has shown that there is no appreciable change in the velocity of a $\beta$ corpuscle during its passage through matter, at any rate for small thicknesses of material ; and this result is supported by results to be described later in this paper. It has been shown theoretically $\dagger$ (and the result is borne out by a comparison of the values obtained for the absorption-coefficient for rays of different velocities) that the penetrating-power of the $\beta$ rays varies as the fourth power of their velocity. Thus if there were any appreciable diminution in the velocity of the rays

* Phil. Mag. vol. xi. p. 779 (June 1906).

$\dagger$ J. J. Thomson, 'Conduction of Electricity though Gases,' p. 315. 
on passing through matter, there should be a marked increase in the coefficient of absorption as the thickness of matter traversed was increased. No such effect was observed, and hence we may assume that, at any rate until the energy of the rays is greatly reduced, there is no appreciable change in their velocity.

As the plan of the experiments included the investigation of elements, many of which could only be obtained in small quantities, the apparatus had to be kept quite small. Its final form was as follows:-The uranium oxide was contained in a depression, 2 mins. deep and $4.5 \mathrm{cms}$. square, in a lead plate A (fig. 1), and covered in with a sheet of aluminium, $0.1 \mathrm{~mm}$. in thickness, in order to cut off all the $\propto$ radiation.

Fig. 1.

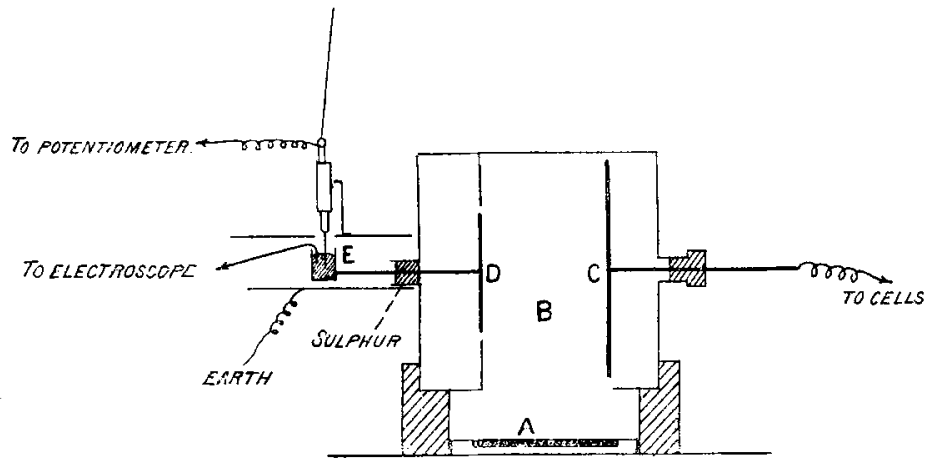

The rays then passed up into the chamber $B$, ionizing the air between two plates $\mathrm{C}$ and $\mathrm{D}, 4 \cdot 2 \mathrm{cms}$. apart. $\mathrm{C}$ was connected to a battery of small storage-cells, and raised to a sufficiently high potential (360 volts) to produce the saturation current through the vessel when the radiation was a maximum. D, $4 \mathrm{cms}$. in diameter, and surrounded by a guard-ring, was connected to a Wilson inclined gold-leaf electroscope through a mercury key $\mathrm{E}$, which could be operated from a distance and by means of which the gold-leaf system could be earthed, left insulated, or charged to a definite potential. The insulations in the gold-leaf system were of sulphur, and the whole was screened from induction effects by earthed metal tubes. In its sensitive position the electroscope was capable of giving 30 scale-divisions per volt, and could be read to one-fifth of a division.

The rate of leak of the electroscope measured the ionization 
in the chamber $B$, and this was in turn a measure of the jntensity of the radiation entering the chamber, and thus the ratio of the leak of the electroscope when the plate $A$ was uncovered (except for the thin aluminium plate used to cut off the $\alpha$ rays) to the leak when the plate $A$ was covered with a thin sheet or layer of the substance under investigation, enabled the coefficient of absorption, $\lambda$, to be calculated, when the thickness of the absorbing material was known.

In almost every case experiments were made with several different thicknesses of the substance under observation, in order to ascertain whether the absorption of the substance was a simple exponential function of the thickness. The logarithms of the intensities of radiation were plotted against the thicknesses of material through which the radiation had passed, and specimen curves so obtained are shown in fig. 2. In the large majority of cases the curve was a straight line, as illustrated by the curves for aluminium and potassium; thus showing that for these snbstances, within the limits of experimental error, the law of absorption was expressed by the relation

$$
\mathrm{I} / \mathrm{I}_{\mathrm{o}}=\epsilon^{-\lambda d} .
$$

In the case of a few of the heavier metals, namely, lead, gold, platinum, tin, silver, and palladium, there was a more or less marked departure from the exponential law, for very small thicknesses of material, as illustrated by the curve for tinfoil (fig. 2, p. 384). McClelland * has shown that this would be the case if an appreciable amount of secondary radiation were produced by the action of the incident $\beta$ rays; and experiments made later upon the above metals showed that there was a considerable amount of return radiation from them. We shall return to this question later. For the present we may state that the value of $\lambda$ employed is that calculated from the straight portion of the curve after $\lambda$ has become constant.

Whenever possible, the element was procured in the form of thin foil. Many, however, were only obtainable as powders. The lighter powders were formed into layers by spreading them out evenly on shallow cardboard trays of different depths; the heavier ones were finely powdered, suspended in chloroform, and allowed to deposit on thin mica sheets. The absorption due to the tray or sheet was of course measured and allowed for.

The thickness of the layer was in every case measured by finding the weight of a known area. (In the case of the

- Scientific Trans. Roy. Soc. Dublin, 1908. 
powders the area was that of the tray or mica sheet.) This method had the advantage of giving the ratio $\lambda / \rho$ directly.

Irig. 2.-Absorption-Curves.

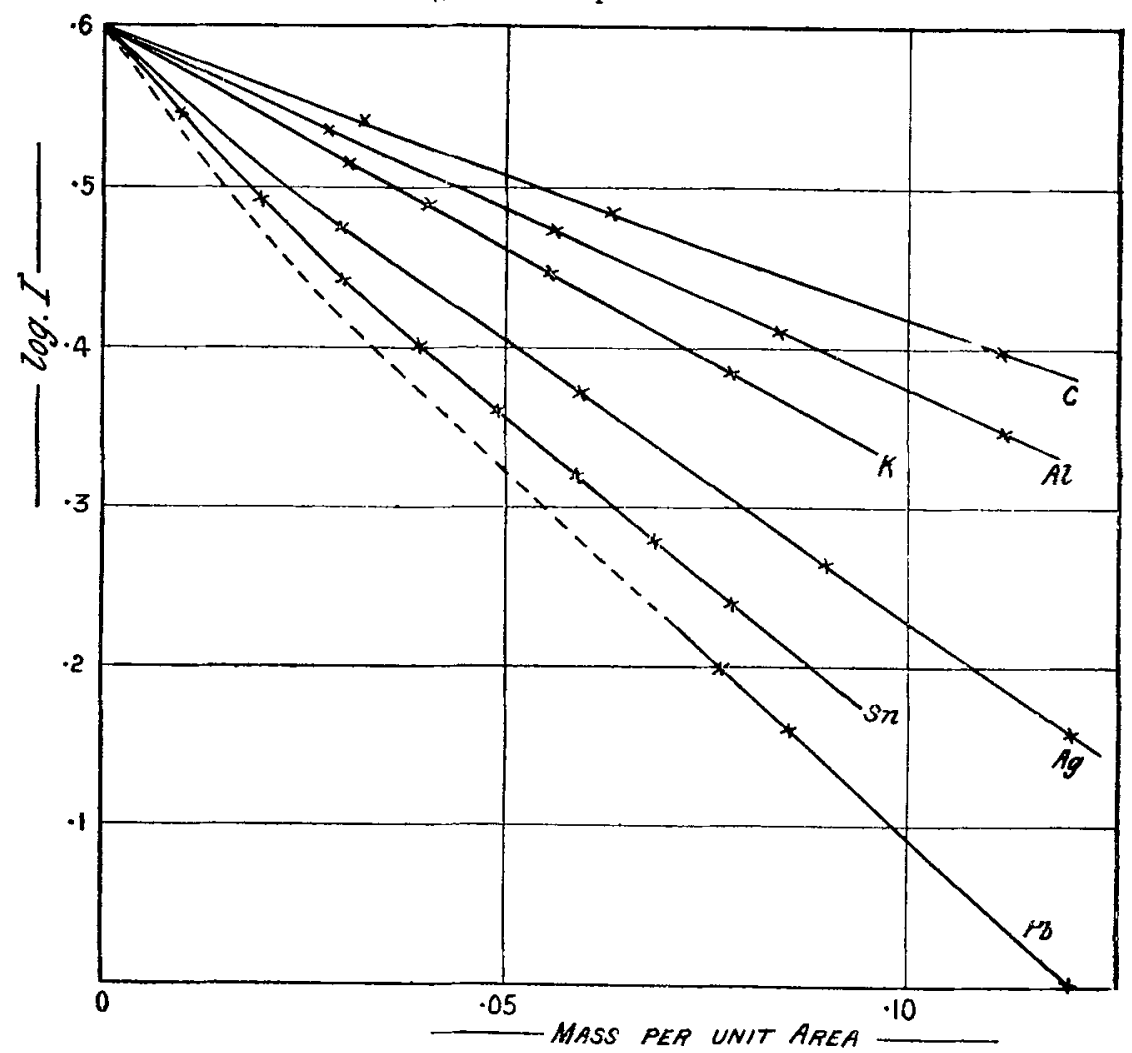

For if $\quad x$ is the thickness of the layer,

$\omega$ its mass, and

$\alpha$ its area,

then $\omega=\alpha \rho x$,

$$
\therefore \quad x=\frac{\omega}{\alpha \cdot \rho}
$$

and $\quad I / I_{0}=\epsilon^{-\lambda x}=\epsilon^{-\frac{\lambda}{\rho} \cdot \frac{\omega}{a}}$.

An accurate knowledge of the density of the particular specimen of the element used was thus rendered unnecessary. For convenience in comparison, thickness in fig. 2 is represented by mass per unit area. 


\section{Alsorption of the Elements.}

Over thirty elements have now been determined, with the results shown in the following table :-

\begin{tabular}{|c|c|c|c|c|c|}
\hline Substance. & At. $\mathbf{W t}$. & $\lambda / \rho$ & Substance. & At. Wt. & $\lambda / \rho$ \\
\hline Boron & 11 & $4 \cdot 65$ & Arsenic & 75 & $8 \cdot 2$ \\
\hline Carbon ............. & 12 & $4 \cdot 4$ & Selenium & 79 & $8 \cdot 65$ \\
\hline Sodium ............ & 23 & $4: 95$ & [Strontium] & 87.5 & 8.5 \\
\hline Magnesium ...... & 244 & $5 \cdot 1$ & Zirconiukn.. & $90 \cdot 7$ & $8 \cdot 3$ \\
\hline Aluminium ...... & 27 & $5 \cdot 26$ & Palladium .......... & 106 & $8 \cdot 0$ \\
\hline Silicon.......... & 28 & $5 \cdot 5$ & 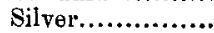 & 108 & $8 \cdot 3$ \\
\hline Phosphorus ...... & 31 & $6 \cdot 1$ & Tin......... & 118 & $9 \cdot 46$ \\
\hline Sulphur .......... & 32 & $6 \cdot 6$ & Antimony & 120 & $9 \cdot 8$ \\
\hline Potassium.......... & 39 & 6.53 & Tellurium. & 126 & $10 \cdot 8$ \\
\hline Calcium... & 40 & $6 \cdot 47$ & Iodine ... & 126 & $10 \cdot 8$ \\
\hline Titanium .. & 48 & $6 \cdot 2$ & [Barium] & 137 & $8 \cdot 8$ \\
\hline Chromiam & 52 & $6 \cdot 25$ & Platinum. & 195 & $9 \cdot 4$ \\
\hline Iron............ & 56 & $6 \cdot 4$ & Gold...$\ldots \ldots \ldots \ldots$ & 197 & $9 \cdot 5$ \\
\hline Cobalt .............. & 59 & $6 \cdot 48$ & Lead. & 207 & $10 \cdot 8$ \\
\hline Copper ............. & $63 \cdot 3$ & $6 \cdot 8$ & [Uranium] ..... & 240 & $10 \cdot 1$ \\
\hline 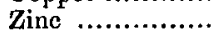 & $65 \cdot 5$ & $6 \cdot 95$ & & & \\
\hline
\end{tabular}

The whole of the above values were obtained from the elements themselves, except in the case of strontium, barium, and uranium, values for which were calculated from the absorption of their oxides, in a way which will be subsequently described. A comparison of these results with thuse of Rutherford shows on the whole a satisfactory agreement, except in the case of tin. The discrepancy here is probably due to the fact that the absorption for tin, as explained above, does not follow a simple exponential law. A calculation of $\lambda$ from the first few points on the curve for tinfoil gives a value very near that of Rutherford.

If now we plot the values of $\lambda / \rho$ given in the above table against the atomic weights, we obtain the curve shown in fig. 3 (p. 386). This curve presents several points of interest. It will be seen at once that the elements arrange themselves very definitely along a series of similar curves. Moreover, these curves correspond closely in extent with the divisions of the Periodic Classification of Chemistry. Boron and carbon stand alone as the only representatives of the first short period. The second short period is represented by a distinct portion of the curve rising from sodium to sulphur. Potassium, the first member of the first long chemical period, is the first point on a fresh portion of the curve, which extends through the whole of the first long period to selenium. Another distinct section of the curve runs from strontium to iodine, thus

Phil.Mag. S. 6. Vol. 12. No. 70. Oct. 1906. 2 C 
coinciding with the second long period; while barium, obviously on still another division of the curve, is the only

Fig. 3.

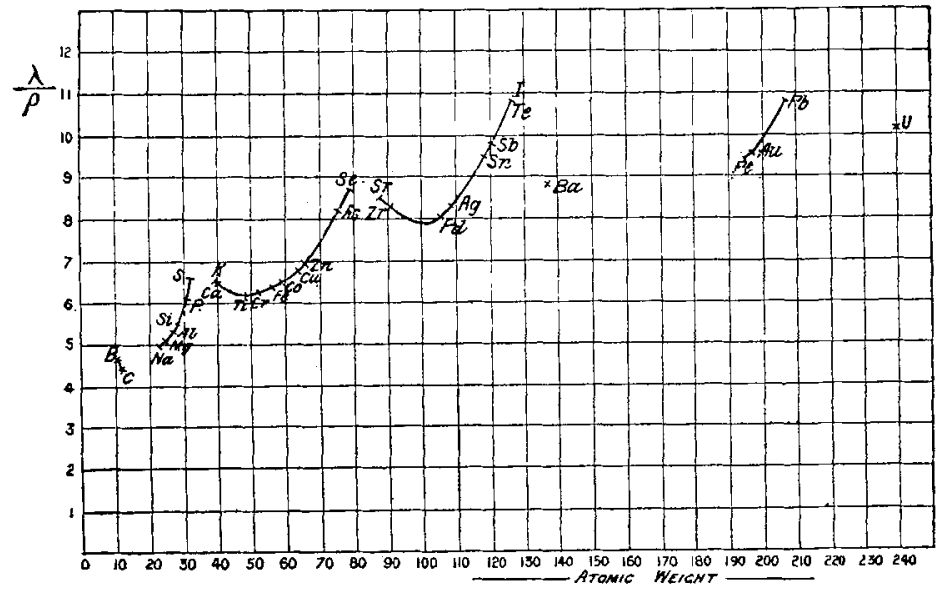

representative of the third. The fourth long period is somewhat poorly represented by platinum, gold, and lead, but sufficient is shown of the curve to prove that this section is distinct from and similar to the previous sections. Uranium is the only representative of the fifth long period, and a fifth portion of the curve. It is interesting to note in passing, that the absorption by uranium of the uranium $\beta$ rays is in no way exceptional, the coefficient of absorption being quite what might be expected from an element of its atomic weight.

Again, the different sections of the curve are very similar in appearance, if we exclude the first short period, which is also somewhat exceptional in character chemically. Each commences with a nearly horizontal portion, and then rises sharply up to a maximum. Practically the whole of the increase occurs in the second half of the long periods.

It will be noticed also that similar elements occur on similar portions of the curve. Thus the alkali and alkali earth metals come at the commencement of the different sections ; the metals of the eighth group come in similar positions near the minima ; while the sulphur, selenium, and tellurium group, and the halogens form the maxima.

In brief, it appears evident that the ratio $\lambda / \rho$, measuring the absorption per corpuscle, is not a constant, but is a periodic function of the atomic weight; the periods corresponding exactly with those of the chemical classification. 
In addition to this periodic variation there is also an increase in the value of the ratio $\lambda / \rho$ with increase of atomic weight. this is clearly brought out by considering groups of similar elements. Thus we bave:-

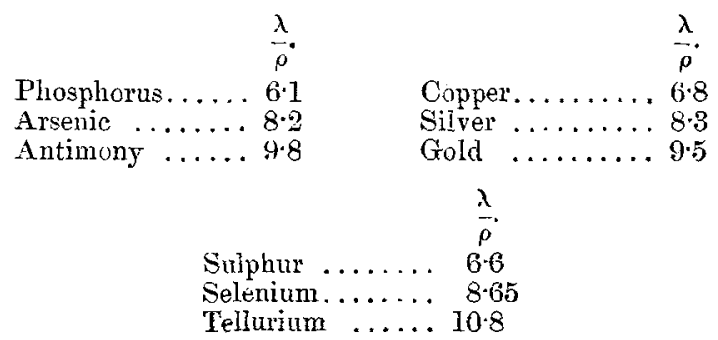

It will thus be seen that passing down any group from elements of lower to elements of higher atomic weight, there is a continuous increase in the value of $\lambda / \rho$. The rate of increase, however, seems to fall off as the atomic weight increases, and is distinctly less rapid after passing the second long period.

\section{Allotropy.}

Several elements occur in two or more distinct forms, differing among themselves in density, appearance, and other physical and chemical properties. It was thought that these different varieties might give slightly different values for the ratio $\lambda / \rho$.

Accordingly a careful comparison was made between pure graphite $(\rho=2 \cdot 25)$ and pure charcoal $(\rho=1 \cdot 85)$ prepared from grape-sugar; but no difference whatever could be detected between the values of $\lambda / \rho$ in the two cases. Similar experiments were made with the different modifications of sulphur, with a similar result. It appears therefore that, whatever the nature of allotropy may be, it does not affect the absorbing power of the atom.

\section{Absorption of Compounds.}

The variation of the absorption per corpuscle with atomic weight having been established, it remained to decide whether the absorption depended upon the state of molecular combination; or, in other words, if the forces which link together the atoms of a compound influence in any way the absorption of the constituent atoms. A considerable number of compounds have been tested with a view to determining this point. 
If we assume that combination does not influence the absorption of the combining atoms, we can easily calculate the value of the ratio $\lambda / \rho$ for a compound from the knowledge of its value for the constituent elements. A comparison between the values so calculated and those obtained by direct experiment will then enable us to test the truth of our assumption.

Let $\mathrm{X}$ and $\mathrm{Y}$ be two elements of atomic weight $m_{1}$ and $m_{2}$ respectively. Since the number of corpuscles in the atom is proportional to the atomic weight, the number of corpuscles in the atoms of $\mathrm{X}$ and $\mathrm{Y}$ respectively will be $k m_{1}$ and $k m_{2}$. Since the absorption per corpuscle is, as previously explained, equal to the ratio $\lambda / \rho$, the absorption per atom of the element $\mathrm{X}$ will be $\frac{\lambda_{1}}{\rho_{1}} k m_{1}$, and that of $\mathrm{Y}, \frac{\lambda_{2}}{\rho_{2}} k m_{2}$.

If, now, the compound is represented by the formula $\mathrm{X}_{a} \mathrm{Y}_{b}$, the absorption per molecule will on our assumption be equal to the sum of the absorptions of the individual atoms, and must therefore equal

$$
a \frac{\lambda_{1}}{\rho_{1}} k m_{1}+b \frac{\lambda_{2}}{\rho_{2}} k m_{2} \text {. }
$$

But the absorption per molecule of a compound will also equal the product of the average absorption per corpuscle multiplied by the number of corpuscles in the molecule; that is, it will equal $\frac{\lambda}{\rho}\left(a k m_{1}+b k m_{2}\right)$, where $\lambda$ and $\rho$ are the values for the compound. Thus equating the two expressions we have

$$
\frac{\lambda}{\rho}=\frac{a \cdot \frac{\lambda_{1}}{\rho_{1}} m_{1}+b \cdot \frac{\lambda_{2}}{\rho_{2}} m_{2}}{a m_{1}+b m_{2}},
$$

which is known when the values of the absorption per corpuscle for the component elements are known.

The following table shows the nature of the agreement between the values calculated from the above formula and the direct experimental values, for fourteen different compounds.

The value of the absorption of oxygen could not be directly determined. The value used in calculating the results in the above table was calculated from the observed absorption of the different oxides, by means of the formula obtained above.

On the whole, the agreement between the observed and the 
Absorption of the $\beta$ Rays from Uranium.

\begin{tabular}{|c|c|c|c|}
\hline \multirow{2}{*}{ Substance. } & \multirow{2}{*}{ Formula. } & \multicolumn{2}{|c|}{$\lambda / \rho$} \\
\hline & & Calculated. & Observed. \\
\hline Magnesium Oxide.... & $\mathrm{MgO}$ & $5 \cdot 6$ & $5 \cdot 5$ \\
\hline Aluminium Oxide.. & $\mathrm{Al}_{2} \mathrm{O}_{3}$ & $5 \cdot 8$ & $5 \cdot 9$ \\
\hline Ferric Oxide.... & $\mathrm{Fe}_{2}^{2} \mathrm{O}_{3}$ & $6 \cdot 4$ & $6 \cdot 4$ \\
\hline Zine Oxide....... & $\mathrm{ZnO}$ & $6 \cdot 8$ & $7 \cdot 3$ \\
\hline Cuprous Oxide... & $\mathrm{Cu}_{2} \mathrm{O}$ & $6 \cdot 8$ & $7 \cdot 1$ \\
\hline Cupric Oxide..... & $\mathrm{CuO}$ & 67 & $6 \cdot 9$ \\
\hline Al'senious Oxide & $\mathrm{As}_{2} \mathrm{O}_{3}$ & $7 \cdot 7$ & $7 \cdot 5$ \\
\hline Ferrous Sulphide...... & Feš & $6 \cdot 7$ & 65 \\
\hline Zine Sulphide & $\mathrm{ZnS}$ & $6 \cdot 8$ & $6 \cdot 8$ \\
\hline Areenious Sulphide ... & $\mathrm{Ag}_{2} \mathrm{~S}_{3}$ & $7 \cdot 5$ & $7 \cdot 5$ \\
\hline Stannous Sulphide ... & Sns & 89 & $8 \cdot 9$ \\
\hline Antimony Sulphide... & $\mathrm{Sb}_{2} \mathrm{~S}_{3}$ & 86 & $8 \cdot 5$ \\
\hline Cuprous Iodide......... & $\mathrm{Cu}_{2} \mathrm{I}_{2}$ & $9 \cdot 4$ & $9 \cdot 1$ \\
\hline Iread Iodide ...... & $\mathrm{PbI}_{2}^{2}$ & $10 \cdot 8$ & $10 \cdot 8$ \\
\hline
\end{tabular}

calculated values is good; in the majority of cases the difference does not exceed the probable experimental error. Hence we may fairly assume that the absorption per molecnle is equal to the sum of the absorptions of the constituent atoms, or in other words that the absorption per corpuscle of an element is not altered when the element enters into a state of combination.

This result was used to calculate the values of the ratio $\lambda / \rho$ for strontium, barium, and uranium. In these cases, where the element to be determined forms a large proportion of the compound, the proportional error introduced is not great. It is clear from the formula, however, that if an element forms only a small proportion by weight of the compound, its absorption will appear as the small difference between two large quantities, and the proportionate error will be greatly increased. Hence it was not possible to use this method to calculate values for the elements of small atomic weight.

\section{Secondary Radiation.}

As mentioned before, McClelland* has shown that even with homogeneous primary $\beta$ rays there would be a departure from the simple exponential law of absorption if any appreciable amount of secondary radiation were produced in the absorbing substance. He also showed that using radium rays the secondary radiation was very considerable. However, as previously explained, in the case of the large majority

* Mcclelland, Scientific Trans. Roy. Soc. Dublin, 1906. 
of substances whose absorption was measured no deviation from the simple exponential law could be detected. This seemed to show that the secondary radiation produced by the $\beta$ rays from uranium was much less in amount than that set up by the radium rays. It was considered desirable to have some direct evidence upon this point.

Owing to the much smaller intensity of the radiation from uranium as compared with that from radium, it was impossible to use any method similar to that of McClelland. The method employed consisted in measuring the ionization between a charged plate and a parallel wire-gauze electrode, due to the uranium radiation; and finding the increase in the ionization when the gauze was backed by a thick plate of the metal under observation. The first reading was a measure of the intensity of the incident radiation $\mathrm{I}_{0}$; the second was proportional to the sum of the incident and the return radiation, that is to $I_{0}+R$, if $R$ is the amount of radiation sent back from the plate. From these two readings therefore we can calculate the value of $R / I_{0}$.

The uranium oxide was contained in a circular depression $2 \mathrm{mms}$. deep and $4 \mathrm{cms}$. in diameter, in the centre of a plate $\mathrm{A}$, $8 \mathrm{cms}$. in diameter, which was kept at a high potential by means of a battery of small storage-cells. The uranium was covered in as before with thin aluminium to cut off all $a$ radiation. The gauze electrode, $8 \mathrm{cms}$. in diameter, was placed parallel to the plate $A$, and connected to the electroscope through a mercury key, in the same manner as in the absorption experiments.

A small amount of primary radiation may escape sideways without striking the npper plate. This, though reckoned in. the incident radiation, does not in reality add its quota to the secondary radiation. However, by placing the electrodes sufficiently close together, we can make this error as small as we please. The measurements were actually made with the plates only $5 \mathrm{mms}$. apart, and it was found that there was very little alteration in the value obtained for the ratio $R / I_{0}$ even if the distance between the plates was increased to twice that amount.

The results obtained showed that, as expected from the absorption-curves, the return radiation was much smaller than that obtained by McClelland with radium rays, and only those substances whose absorption-curves departed from the exponential law gave very appreciable amounts. The following briof table gives a comparison of the results obtained by this method for uranium rays, with McClelland's results for radinm. 
Absorption of the $\beta$ Rays from Uranium.

\begin{tabular}{|c|c|c|}
\hline \multirow{2}{*}{ Substance. } & \multicolumn{2}{|c|}{$\mathrm{R} / \mathrm{I}_{0} \times 100$} \\
\hline & Radium. & Uranium. \\
\hline Lead ........... & 49 & 15 \\
\hline Gold ........... & ... & $14 \cdot 5$ \\
\hline Platinum ........ & 47 & $14: 5$ \\
\hline $\operatorname{Tin} \quad \ldots \ldots \ldots \ldots . . . . .$. & $42 \cdot 5$ & 12 \\
\hline Copper ....... & 36 & 4 \\
\hline Aluminium ..... & 24 & very small. \\
\hline
\end{tabular}

It will be at once seen that the values for uranium are considerably smaller than those for radium rays, and the ratio also seems to decrease more rapidly with the atomic weight for the rays from the former substance.

These differences may possibly be due in part to the presence in the radium radiation of comparatively slowly moving $\beta$ rays. Becquerel has shown that the amount of secondary radiation produced decreases rapidly as the velocity of the priniary rays is increased. It seems possible therefore that a large proportion of the secondary radiation due to the radium rays may be set up by the more slowly moving portions of the rays, while the more penetrating portion corresponding to the uranium rays in velocity may produce comparatively little.

Whatever the cause, both the absorption-curves and the direct experiments seem to show that the secondary radiation set up by the uranium rays is distinetly less in amount than that produced by radium radiation.

No attempt has been made to calculate what McClelland has called the true coefficient of absorption for the rays.

McClelland* has shown that, if secondary radiation is set up, the true coefficient of absorption $\mu$ of the rays is connected with the coefficient $\lambda$ as previously defined, by the equation

$$
\lambda=\mu \sqrt{1-k},
$$

where $k$ is the proportion of absorbed energy which is restored to the stream of rays as secondary radiation. He further shows that this quantity $k$ is connected with the total return

* McClelland, loc. cit. 
radiation from a thick plate of the substance by the relation

$$
\mathrm{R} / \mathrm{I}_{\mathrm{o}}=\frac{1-\frac{1}{2} k-\sqrt{1-k}}{\frac{1}{2} k},
$$

or if we denote $R / I_{0}$ by the letter $p$

$$
k=\frac{4 p}{(p+1)^{2}} .
$$

We are thus enabled to calculate $\mu$ when $p$ and $\lambda$ are known.

The secondary radiation in the case of uranium rays was, however, so small, that the errors of observation were so great as to outweigh any theoretical advantage there might possibly be in the use of the "true" coefficient $\mu$, instead of the coefficient $\lambda$. The return radiation $R$ was obtained as the difference of two quantities, $I_{0}$ and $I_{0}+R$, which in most cases were nearly equal. The proportionate error in $R$ therefore was very great. Thus even in the case of lead, an error of only 1 per cent. in measuring the intensity of $I_{0}$, or $I_{0}+R$, would produce an error of 7 per cent. in the value of $p$, while in the case of copper the error would be as much as 25 per cent. As, owing to the smallness of the radiation from uranium, it was certainly not possible to ensure an accuracy greater than 1 per cent. in the measurement of the intensities of the rays, it was considered preferable to retain the directly measured coefficient $\lambda$.

No great amount of error is introduced in this way, and the general results of the experiment are quite unaltered. In the majority of cases the secondary radiation was almost inappreciable, and $\lambda$ therefore very nearly equal to $\mu$. Substituting the values of $p$ actually obtained, we have for aluminium, $\mu$ is equal to $\lambda$; for copper, $\mu$ equals $1.08 \lambda$; while for tin, gold, platinum, and lead, which show the maximum effect, $\mu$ is equal to $1 \cdot 3 \lambda$. The effect, moreover, is roughly proportional to the atomic weight.

The effect of substituting $\mu$ for $\lambda$ in the curve of fig. 3 would therefore not affect the general appearance of the curve, nor the general results deduced therefrom. Its only effect would be to slightly increase the slope of the later portions, and thus somewhat increase the rate of increase of the absorption with atomic weight.

In conclusion I wish to express my best thanks to Prof. J. J. Thomson for his kindness in suggesting the subject of this research, and also for much kindly interest during the course of the experiments.

Cavendish Laboratory, Cambridge, August 3rd, 1906. 